$$
\begin{aligned}
\pi^{-1} \int_{-\pi}^{\pi}\left|p_{n}^{\prime}\left(e^{i \theta}\right)\right|^{2} d \theta & \leqq(2 \pi)^{-1} \int_{-\pi}^{\pi}\left|q_{n}^{\prime}\left(e^{i \theta}\right)\right|^{2} d \theta+(2 \pi)^{-1} \int_{-\pi}^{\pi}\left|p_{n}^{\prime}\left(e^{i \theta}\right)\right|^{2} d \theta \\
& =\sum_{\nu=0}^{n}\left(\nu^{2}+(n-\nu)^{2}\right)\left|\alpha_{\nu}\right|^{2} .
\end{aligned}
$$

The greatest of the numbers $\nu^{2}+(n-\nu)^{2}, \nu=1, \cdots, n$, is $n^{2}$. Therefore

$$
\pi^{-1} \int_{-\pi}^{\pi}\left|p_{n}^{\prime}\left(e^{i \theta}\right)\right|^{2} d \theta \leqq n^{2} \sum_{\nu=0}^{n}\left|\alpha_{\nu}\right|^{2}
$$

which was the assertion.

For $p_{n}(z)=\left(z^{n}+1\right) / 2$ the sign of equality holds in (8).

New York City

\title{
A NEW FORMULA FOR INVERSE INTERPOLATION
}

\section{HERBERT E. SALZER}

This paper is devoted to the derivation of a formula for inverse interpolation in a table of equally spaced arguments. The resulting formula (5) is more concise and convenient than those in existence. It involves neither differences nor polynomial coefficients other than small powers. In use it will be found much simpler and quicker than those given by Davis, Aitken, Steffensen and Milne-Thomson. In a sense, it is the analogue of the Lagrangian formula for direct interpolation without differences (that is, in terms of the tabular entries only) if the usual expression (right member of (1) below) is rearranged in terms of powers of the argument $p$.

Lagrange's general interpolation formula is

$$
f(x)=\sum_{\nu=0}^{\nu=k} \frac{P_{\nu}(x)}{P_{\nu}\left(a_{\nu}\right)} f\left(a_{\nu}\right), \quad \text { where } \quad P_{\nu}(x)=\frac{1}{\left(x-a_{\nu}\right)} \prod_{i=0}^{i=k}\left(x-a_{i}\right) .
$$

For equally spaced arguments at interval $h_{0}$ after suitable relabelling of the arguments $a_{i}$, Lagrange's formula becomes

$$
f_{p}=\sum_{i=-[(n-1) / 2]}^{i=[n / 2]} L_{i}^{(n)}(p) f_{i}
$$

Received by the editors January 1, 1944. 
where $f_{x}$ denotes $f\left(a_{0}+x h\right),[x]$ denotes the largest integer in $x$, and $L_{i}^{(n)}(p)$ are well known polynomials in $p$ of the $(n-1)$ th degree. Making use of the relation

$$
\sum_{i=-[(n-1) / 2]}^{i=[n / 2]} L_{i}^{(n)}(p) \equiv 1
$$

we can rewrite (1) as

$$
\frac{p}{f_{p}-f_{0}}=1 / \sum_{i=-[(n-1) / 2]}^{i=[n / 2]} \frac{L_{i}^{(n)}(p)}{p}\left(f_{i}-f_{0}\right) .
$$

Since $L_{0}^{(n)}(p) / p$ is not present in the summation, the denominator of the right-hand side of (3) is still a polynomial in $p$.

Now we employ an expansion due to Bürmann which states that if $\psi(z)=(z-a) /(\phi(z)-b)$, then

$$
f(z)=f(a)+\sum_{m=1}^{k-1} \frac{[\phi(z)-b]^{m}}{m !} \frac{d^{m-1}}{d a^{m-1}}\left[f^{\prime}(a)\{\psi(a)\}^{m}\right]+R_{k} .
$$

Consider the equation

$$
\frac{p-a}{f_{p}-f_{0}}=1 / \sum_{i=-[(n-1) / 2]}^{i=[n / 2]} \frac{L_{i}^{(n)}(p)}{p}\left(f_{i}-f_{0}\right) .
$$

Letting

$z=p, \quad \phi(p)=f_{p}, \quad b=f_{0}, \quad \psi(p)=1 / \sum_{i=-[(n-1) / 2]}^{i=[n / 2]} \frac{L_{i}^{(n)}(p)}{p}\left(f_{i}-f_{0}\right)$ and $f(p)=p$, we obtain, by Bürmann's expansion,

$$
\begin{aligned}
p=a+ & \sum_{m=1}^{k-1} \frac{\left(f_{p}-f_{0}\right)^{m}}{m !} \\
& \frac{d^{m-1}}{d a^{m-1}}\left(1 / \sum_{i=-[(n-1) / 2]}^{i=[n / 2]} \frac{L_{i}^{(n)}(a)}{a}\left(f_{i}-f_{0}\right)\right)^{m}+R_{k}^{\prime} .
\end{aligned}
$$

To obtain $p$ as an explicit function of $f_{p}$ and the $f_{i}$ 's according to its implicit definition in (1) or (3), we take the limit of $\left(4^{\prime}\right)$ as $a \rightarrow 0$ and arrive at the following expansion:

$$
p=\sum_{m=1}^{k-1} \frac{\left(f_{p}-f_{0}\right)^{m}}{m !}
$$

$$
\left[\frac{d^{m-1}}{d a^{m-1}}\left(1 / \sum_{i=-[(n-1) / 2]}^{i=[n / 2]} \frac{L_{i}^{(n)}(a)}{a}\left(f_{i}-f_{0}\right)\right)^{m}\right]_{a=0}+R_{k}^{\prime \prime} .
$$


Corresponding to the $n$-point formulas for direct interpolation, $n$ ranging from 3 to 7 , we define quantities $r, s, t, u, v$ and $w$ thus:

$$
\begin{aligned}
& r=\frac{2\left(f_{p}-f_{0}\right)}{f_{1}-f_{-1}}, \\
& s=\frac{f_{1}-2 f_{0}+f_{-1}}{f_{1}-f_{-1}}, \\
& t=u=v=w=0 .
\end{aligned}
$$

$$
\begin{aligned}
& r=\frac{6\left(f_{p}-f_{0}\right)}{-f_{2}+6 f_{1}-3 f_{0}-2 f_{-1}}, \\
& s=\frac{3\left(f_{1}-2 f_{0}+f_{-1}\right)}{-f_{2}+6 f_{1}-3 f_{0}-2 f_{-1}}, \\
& t=\frac{f_{2}-3 f_{1}+3 f_{0}-f_{-1}}{-f_{2}+6 f_{1}-3 f_{0}-2 f_{-1}}, \\
& u=v=w=0 .
\end{aligned}
$$

5-point

$$
\begin{aligned}
& r=\frac{24\left(f_{p}-f_{0}\right)}{-2 f_{2}+16 f_{1}-16 f_{-1}+2 f_{-2}}, \\
& s=\frac{-f_{2}+16 f_{1}-30 f_{0}+16 f_{-1}-f_{-2}}{-2 f_{2}+16 f_{1}-16 f_{-1}+2 f_{-2}}, \\
& t=\frac{2\left(f_{2}-2 f_{1}+2 f_{-1}-f_{-2}\right)}{-2 f_{2}+16 f_{1}-16 f_{-1}+2 f_{-2}}, \\
& u=\frac{f_{2}-4 f_{1}+6 f_{0}-4 f_{-1}+f_{-2}}{-2 f_{2}+16 f_{1}-16 f_{-1}+2 f_{-2}}, \\
& v=w=0 .
\end{aligned}
$$

6-point

$$
r=\frac{120\left(f_{p}-f_{0}\right)}{4 f_{3}-30 f_{2}+120 f_{1}-40 f_{0}-60 f_{-1}+6 f_{-2}},
$$$$
s=\frac{5\left(-f_{2}+16 f_{1}-30 f_{0}+16 f_{-1}-f_{-2}\right)}{4 f_{3}-30 f_{2}+120 f_{1}-40 f_{0}-60 f_{-1}+6 f_{-2}},
$$$$
t=\frac{5\left(-f_{3}+7 f_{2}-14 f_{1}+10 f_{0}-f_{-1}-f_{-2}\right)}{4 f_{3}-30 f_{2}+120 f_{1}-40 f_{0}-60 f_{-1}+6 f_{-2}}
$$$$
u=\frac{5\left(f_{2}-4 f_{1}+6 f_{0}-4 f_{-1}+f_{-2}\right)}{4 f_{3}-30 f_{2}+120 f_{1}-40 f_{0}-60 f_{-1}+6 f_{-2}},
$$$$
v=\frac{f_{3}-5 f_{2}+10 f_{1}-10 f_{0}+5 f_{-1}-f_{-2}}{4 f_{3}-30 f_{2}+120 f_{1}-40 f_{0}-60 f_{-1}+6 f_{-2}}
$$

$w=0$.

7-point

$$
\begin{aligned}
r & =\frac{720\left(f_{p}-f_{0}\right)}{12 f_{3}-108 f_{2}+540 f_{1}-540 f_{-1}+108 f_{-2}-12 f_{-8}}, \\
s & =\frac{2\left(2 f_{8}-27 f_{2}+270 f_{1}-490 f_{0}+270 f_{-1}-27 f_{-2}+2 f_{-8}\right)}{12 f_{3}-108 f_{2}+540 f_{1}-540 f_{-1}+108 f_{-2}-12 f_{-8}}, \\
t & =\frac{15\left(-f_{3}+8 f_{2}-13 f_{1}+13 f_{-1}-8 f_{-2}+f_{-8}\right)}{12 f_{8}-108 f_{2}+540 f_{1}-540 f_{-1}+108 f_{-2}-12 f_{-8}}, \\
u & =\frac{5\left(-f_{3}+12 f_{2}-39 f_{1}+56 f_{0}-39 f_{-1}+12 f_{-2}-f_{-8}\right)}{12 f_{3}-108 f_{2}+540 f_{1}-540 f_{-1}+108 f_{-2}-12 f_{-8}}, \\
v & =\frac{3\left(f_{3}-4 f_{2}+5 f_{1}-5 f_{-1}+4 f_{-2}-f_{-3}\right)}{12 f_{3}-108 f_{2}+540 f_{1}-540 f_{-1}+108 f_{-2}-12 f_{-3}}, \\
w & =\frac{f_{3}-6 f_{2}+15 f_{1}-20 f_{0}+15 f_{-1}-6 f_{-2}+f_{-8}}{12 f_{3}-108 f_{2}+540 f_{1}-540 f_{-1}+108 f_{-2}-12 f_{-8}}
\end{aligned}
$$

Then in every case (4) assumes the form 


$$
\begin{aligned}
p= & r-r^{2} s+r^{3}\left(2 s^{2}-t\right)+r^{4}\left(-5 s^{3}+5 s t-u\right) \\
& +r^{5}\left(14 s^{4}-21 s^{2} t+3 t^{2}+6 s u-v\right) \\
& +r^{6}\left(-42 s^{5}+84 s^{3} t-28 s t^{2}-28 s^{2} u\right. \\
& +7 t u+7 s v-w)+\cdots .
\end{aligned}
$$

When (5) is employed for interpolation for a number of values within the same interval, the only variable is $r^{k}$.

From (5) which gives $p(r)$, it is immediately apparent that for numerical integration of the inverse function we obtain

$$
\int_{f_{0}}^{f_{p}} x\left(f_{t}\right) d\left(f_{t}\right)=\left(f_{p}-f_{0}\right)\left(a_{0}+(h / r) \int p(r) d r\right),
$$

while for the $m$ th derivative of the inverse function we have

$$
x^{(m)}\left(f_{p}\right)=\left(h r^{m} /\left(f_{p}-f_{0}\right)^{m}\right) p^{(m)}(r) .
$$

Mathematical Tables Project, National Bureau of Standards, New York City 